\title{
Neural gain control measured through cortical gamma oscillations is associated with sensory sensitivity
}

\author{
Elena V. Orekhova ${ }^{1,2,3}$ @ | Tatiana A. Stroganova ${ }^{2,3}$ | Justin F. Schneiderman ${ }^{4,5}$ ( ) | \\ Sebastian Lundström ${ }^{1}$ | Bushra Riaz ${ }^{4}$ | Darko Sarovic ${ }^{1}$ | Olga V. Sysoeva ${ }^{2,3}$ | \\ Georg Brant ${ }^{5}$ | Christopher Gillberg ${ }^{1}$ | Nouchine Hadjikhani ${ }^{1,6}$ (1)
}

${ }^{1}$ Gillberg Neuropsychiatry Centre (GNC), University of Gothenburg, Gothenburg, Sweden

${ }^{2}$ Moscow State University of Psychology and Education, Center for Neurocognitive Research (MEG Center), Moscow, Russia

${ }^{3}$ Autism Research Laboratory, Moscow State University of Psychology and Education, Moscow, Russia

${ }^{4}$ Department of Clinical Neurophysiology, University of Gothenburg, Institute of Neuroscience \& Physiology, Gothenburg, Sweden

${ }^{5}$ Chalmers University of Technology and MedTech West, Gothenburg, Sweden

${ }^{6} \mathrm{MGH} / \mathrm{MIT} / \mathrm{HST}$ Martinos Center for Biomedical Imaging, Harvard Medical School, Charlestown, Massachusetts

\section{Correspondence}

Elena V. Orekhova, MEG Centre, Moscow State University of Psychology and Education, Shelepihinskaja embankment 2a, 123290

Moscow, Russia.

Email: orekhova.elena.v@gmail.com

Funding information

Charity Foundation "Vykhod" ("Way Out");

LifeWatch - Niclas Öberg Foundation;

Swedish Childhood Cancer Foundation, Grant/

Award Number: MT2014-0007; Swedish

Research Council, Grant/Award Number:

621-2012-3673; Knut and Alice Wallenberg

Foundation, Grant/Award Number:

2014.0102; Russian Academic Excellence

Project '5-100'; HSE Basic Research Program;

Russian Science Foundation (RSF), Grant/

Award Number: 14-35-00060; Torsten

Soderberg Foundation, Grant/Award Number: M240/13

\begin{abstract}
Gamma oscillations facilitate information processing by shaping the excitatory input/output of neuronal populations. Recent studies in humans and nonhuman primates have shown that strong excitatory drive to the visual cortex leads to suppression of induced gamma oscillations, which may reflect inhibitory-based gain control of network excitation. The efficiency of the gain control measured through gamma oscillations may in turn affect sensory sensitivity in everyday life. To test this prediction, we assessed the link between self-reported sensitivity and changes in magnetoencephalographic gamma oscillations as a function of motion velocity of high-contrast visual gratings. The induced gamma oscillations increased in frequency and decreased in power with increasing stimulation intensity. As expected, weaker suppression of the gamma response correlated with sensory hypersensitivity. Robustness of this result was confirmed by its replication in the two samples: neurotypical subjects and people with autism, who had generally elevated sensory sensitivity. We conclude that intensity-related suppression of gamma response is a promising biomarker of homeostatic control of the excitation-inhibition balance in the visual cortex.
\end{abstract}

\section{KEYWORDS}

autism spectrum disorders, gamma oscillations, magneto-encephalography, response gain control, sensory sensitivity, visual motion

\section{1 | INTRODUCTION}

The balance between excitation and inhibition (E-I balance) in neural networks orchestrates neural activity in space and time, and is important for cortical functioning (Dorrn, Yuan, Barker, Schreiner, \& Froemke, 2010; Isaacson \& Scanziani, 2011; Xue, Atallah, \& Scanziani, 2014). Activity of the excitatory $(\mathrm{E})$ and inhibitory $(\mathrm{I})$ neurons is fine-balanced in the normal brain and this balance is disrupted in epilepsy (Dehghani et al., 2016) and neurodevelopmental disorders, such as, for example, autism and schizophrenia (LeBlanc \& Fagiolini, 2011; Lee, Lee, \& Kim, 2017; Nelson \& Valakh, 2015; Rubenstein \& Merzenich, 2003).

Animal findings promote the discoveries of new drugs aimed to restore the neural E-I balance in the patients with brain and mental health disorders (Lee et al., 2017; Tu et al., 2017); however, there are still considerable challenges in testing them in clinical trials. A lack of quantifiable noninvasive measures of the $\mathrm{E}-\mathrm{I}$ balance in the human brain precludes stratification of heterogeneous patient populations according to the distinct E-I balance subtypes, and hinders assessment 
of the treatment efficacy (Ecker, Spooren, \& Murphy, 2013; Levin \& Nelson, 2015).

The stimulus-induced high-frequency magneto-encephalography (MEG)/EEG gamma oscillations (30-100 Hz) have attracted considerable attention as a putative noninvasive indicator of an altered $\mathrm{E}-\mathrm{I}$ balance in human cortex (Levin \& Nelson, 2015; Nelson \& Valakh, 2015). Gamma oscillations are generated by populations of interconnected excitatory and inhibitory neurons and are intimately related to the balance between inhibitory and excitatory neurotransmission (Buzsaki \& Wang, 2012; Vinck, Womelsdorf, \& Fries, 2013). However, the current attempts to define a single parameter of human gamma response that would accurately capture the E-I balance have led to ambiguous results (Cousijn et al., 2014; Edden, Muthukumaraswamy, Freeman, \& Singh, 2009; Perry, Brindley, Muthukumaraswamy, Singh, \& Hamandi, 2014).

In a previous study we put forward the idea that it is possible to estimate efficiency of the E-I balance regulation in the visual cortex through probing input-output gain in the strength of the visual gamma oscillations recorded by MEG (Orekhova et al., 2018). Response gain control is a basic property of neural networks that works to both amplify the neuronal responses to weak sensory signals and to saturate/suppress these responses under conditions of excessive input (e.g., Peirce, 2007). The strength of visually induced gamma oscillations is controlled in accord with this mechanism, in both animals (Jia, Smith, \& Kohn, 2011; Jia, Xing, \& Kohn, 2013; Roberts et al., 2013; Salelkar, Somasekhar, \& Ray, 2018) and humans (Orekhova et al., 2018), wherein a gradual increase in excitatory drive elicits an increase in gamma response power up to a certain "transition" point, and a stimulation intensity past that point leads to suppression of the gamma response.

According to modeling studies, suppression of the oscillatory gamma response at high intensities of excitatory drive is caused by over-excitation of the I-neurons resulting in the loss of neural synchrony (Borgers \& Kopell, 2005; Borgers \& Walker, 2013; Cannon et al., 2014). Computational models further suggest that the gamma suppression is substantially reduced when the excitation of the Eneurons is disproportionally higher than that of the I-neurons (i.e., in the case of a high E-I ratio) (Borgers \& Kopell, 2005). Indeed, optogenetic research in animals demonstrated that gamma oscillations are particularly powerful when the high excitation of excitatory neurons is not properly balanced by inhibition (Yizhar et al., 2011). In regard to humans, these considerations imply that the brain of individuals exhibiting weaker suppression of the visual MEG gamma response is characterized by less efficient inhibitory-based capacity to down-regulate the rising excitation, that is, by an E-I ratio shifted toward excitation (see Orekhova et al., 2018, for discussion).

If suppression of the visual gamma response does reflect the capacity to regulate the $\mathrm{E}-\mathrm{I}$ balance in the visual cortex, this phenomenon should have behavioral manifestations. On the behavioral level, the enhanced neural excitability of sensory cortices is associated with heightened or aversive reactions to intensive sensory input. Indeed, subjective discomfort associated with intensive visual stimulation correlates positively with hemodynamic responses in the visual cortex (Bargary, Furlan, Raynham, Barbur, \& Smith, 2015; Haigh et al., 2013). Moreover, people with neurological or neuropsychiatric disorders characterized by overt clinical symptoms of elevated neuronal excitability, such as migraine with visual aura (Boulloche et al., 2010; Maniyar,
Sprenger, Schankin, \& Goadsby, 2014; O'Hare \& Hibbard, 2016) or epilepsy (Van Campen et al., 2015) often suffer from sensory hypersensitivity. It seems plausible that the atypically strong cortical responses observed in people reporting sensory hypersensitivity are caused by deficiency of the gain control mechanisms that balance excitation and inhibition in the sensory cortices. Therefore, we predicted that reduced suppression of gamma response at high intensities of visual input would be associated with enhanced sensory sensitivity in everyday life.

Here, we sought to test this prediction in two independent samples of subjects: neurotypical individuals (NT) and high-functioning individuals with autism spectrum disorder (ASD). A large proportion of people with ASD are hypersensitive to environmental stimuli of different modalities. Considerable variations in sensitivity to sensory events are also present in the general population (Horder, Wilson, Mendez, \& Murphy, 2014; Little, Dean, Tomchek, \& Dunn, 2017), correlate with autistic features (Horder et al., 2014; Robertson \& Simmons, 2013), and share with them a common genetic basis (Taylor et al., 2018). Therefore, we expected that the similar neuro-behavioral association should characterize both groups. To test this prediction, we assessed behavioral sensory sensitivity using the Adolescent/Adult Sensory Profile (A/ASP) questionnaire (Brown \& Dunn, 2002) and measured velocity-related suppression of the visual MEG gamma response in adults with and without ASD. Since we used moving visual stimuli, we expected to find the most prominent neurobehavioral correlations for the visual modality, and particularly for sensitivity to visual motion.

\section{2 | MATERIALS AND METHODS}

\section{1 | Participants}

Twenty individuals (1 female) with ASD were included in the study, these were drawn from two study groups that have been described elsewhere (Davidsson et al., 2017; Helles, Gillberg, Gillberg, \& Billstedt, 2015). Briefly, 14 individuals had been assigned an ASDdiagnosis at three different occasions by structured clinical interviews. The remaining five individuals had been assigned an ASD-diagnosis at the Clinical Neuropsychiatry Centre in Gothenburg and then via a parental interview. One individual with ASD was recruited via advertisement, whose health journals were scrutinized and reviewed by a senior child and adolescent psychiatrist in order to verify the diagnosis. Nineteen "neuro-typical" (NT, all males) participants were recruited via advertisement. The NT participants underwent a brief screening focusing on neurological and psychiatric disorders in order to rule out psychopathology. To assess cognitive ability the Wechsler Adult Intelligence Scale, fourth edition (WAIS-IV) was used (for a few subjects in the ASD-group WAIS-III data was used). Individuals with an IQ below 80 were excluded. The NT and ASD group did not differ significantly in either age (ASD: $18.8-50.0$ years, mean $=31.1, S D=$ 7.9; NT: $19.2-40.1$ years, mean $=27.3, S D=6.4 ; p>.1$ ) or general IQ (ASD: 78-140, mean $=108.8, S D=15.7 ;$ NT: 96-135, mean = $114.0, S D=11.5 ; p>.2$ ). The study has ethical approval from the regional ethical review board in Gothenburg (DNR: 552-14). Participants followed the informed consent procedure and were repeatedly given the option to discontinue their participation in the study. 


\subsection{Assessment of sensory function}

All the subjects filled in the Adolescent/Adult Sensory Profile questionnaire (A/ASP) (Brown \& Dunn, 2002). This instrument combines information about sensory processing into four categories: "Low Registration," "Sensation Seeking," "Sensory Sensitivity," and "Sensation Avoiding." Here, we were interested in the Sensory Sensitivity scale that measures passive behavioral responses that characterize an individual's sensitivity to environmental events, such as noticing behaviors, distractibility, and discomfort with sensory stimuli.

The A/ASP also allows assessment of the "Neurological Thresholds" by combining the items across categories. Combined scores on the "Sensory Sensitivity" and "Sensation Avoiding" categories constitute the "Low Neurological Threshold" (called below "Low Threshold") that measures a person's notice of, or annoyance with, sensory stimuli. This "Low Threshold" category can be pooled for sensory modalities, as well as calculated separately for sensory/behavioral domains. Here we were mainly interested in the "Low Threshold" for the visual modality.

Since we used moving visual stimuli in the present study, the velocity-related suppression of gamma response might most closely reflect subject's sensitivity to the moving visual stimuli. Therefore, we also introduced the "Visual Motion Sensitivity" scale by combined two A/ASP items that measured subject's discomfort associated with intensive visual motion [i.e., Item 22: "I am bothered by unsteady or fast moving visual images in movies or TV"; Item 25: "I become bothered when I see lots of movement around me (for example, at a busy mall, parade, carnival)"].

\section{3 | Experimental task}

To measure gamma, we applied an experimental paradigm that has been shown to induce reliable MEG gamma responses in the visual cortex in our previous studies (Orekhova et al., 2015, 2018; Stroganova et al., 2015). The schematic representation of the experimental paradigm is given in Figure 1. The stimuli were generated using Presentation software (Neurobehavioral Systems Inc., The United States) and presented using a FL35 LED DPL gamma-corrected projector with $1,920 \times 1,080$ screen resolution and $120 \mathrm{~Hz}$ refresh rate. They consisted of black and white sinusoidally modulated annular gratings with a spatial frequency of 1.66 cycles per degree of visual angle and an outer diameter of $18^{\circ}$ of visual angle. The gratings appeared in the center of a screen over a black background and drifted to the central point at velocities of $1.2,3.6$, or $6.0^{\circ} / \mathrm{s}$, (which approximately corresponds to temporal frequencies of 2,6 , and $10 \mathrm{~Hz}$, respectively); hereafter, we respectively refer to these three velocities as "slow," "medium," and "fast." Each trial began with the presentation of a white fixation cross in the center of the display over a black background for $1,200 \mathrm{~ms}$ that was followed by the grating that drifted for 1,200-3,000 ms and then stopped. The participants were instructed to respond to the termination of motion with a button press. If no response occurred within $1 \mathrm{~s}$, the grating was substituted by a discouraging message "too late!" that remained on the screen for $2,000 \mathrm{~ms}$, after which a new trial began. Error trials (misses or responses that occurred $<150 \mathrm{~ms}$ after the stop) were excluded from

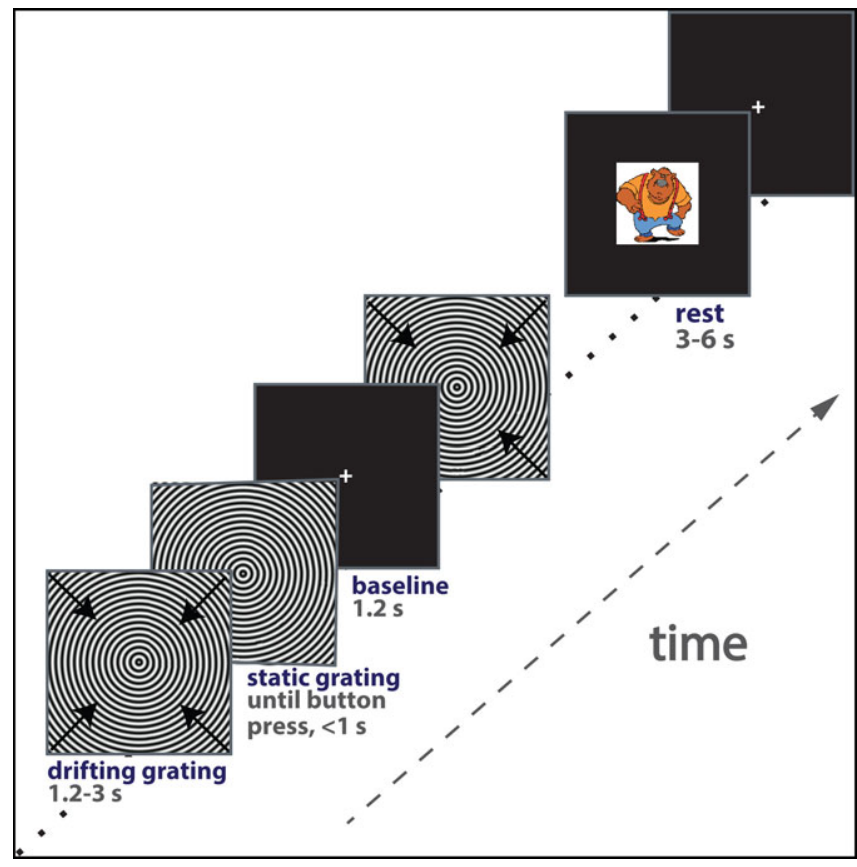

FIGURE 1 Experimental design. Each trial began with presentation of a fixation cross that was followed by an annular grating drifting inward for 1.2-3 s at one of the three velocities: $1.2,3.6,6.0^{\circ} / \mathrm{s}$. Hereafter, we referred to these velocities as "slow," "medium," and "fast." Arrows indicate direction of the motion. Participants responded to the termination of motion with a button press. Short (3-6s) animated cartoon characters were presented randomly between every 2-5 stimuli to sustain vigilance and reduce visual fatigue [Color figure can be viewed at wileyonlinelibrary.com]

the MEG analysis. Stimuli were presented in three experimental blocks in a random order resulting in 90 repetitions of each stimulus type. The luminance of the screen measured at the position of the observer's eyes was 53 Lux during the stimulation and 2.5 Lux during the inter-stimulus interval. Short (3-6 s) animated cartoon characters were presented randomly between every 2-5 stimuli to increase vigilance and minimize fatigue.

\subsection{Eye movements recording and analysis}

Differences in oculomotor behavior could potentially affect the induced gamma responses and interfere with the effects of condition and experimental group. For example, it has been shown that microsaccades performed by subjects during presentation of visual stimuli induce gamma activity (Yuval-Greenberg, Tomer, Keren, Nelken, \& Deouell, 2008). Differences in fixation position also could affect the gamma responses induced by the moving visual stimuli. In order to assess the effects of these factors on our MEG findings, we recorded bilateral eye movements in our participants using a MEG compatible eyetracker (EyeLink 1,000). Five subjects (1 NT and 4 ASD) were excluded from the eye movement analysis because of technical artifacts. In the remaining subjects (16 ASD and $18 \mathrm{NT}$ ), we calculated microsaccade rate during the entire analysis interval ( -1 to $1.2 \mathrm{~s}$ relative to the stimulus onset) as the number of microsaccades per second. We also calculated the average probability of microsaccade occurrence (microsaccades per time sample) during the prestimulus 
interval ( -1 to $0 \mathrm{~s})$ and during the visual stimulation ( 0.3 to $1.2 \mathrm{~s}$ interval). The distance between the fixation position from the middle of the screen ("median deviation") was furthermore estimated separately for prestimulus and stimulation intervals. Details of the eye movement analysis are provided in Supporting Information Methods.

\section{5 | MEG recording}

MEG was recorded at the NatMEG Centre (The Swedish National Facility for Magnetoencephalography, Karolinskalnstitutet, Stockholm) using 306-channel system (ElektaNeuromag TRIUX). The data was recorded with a band-pass filter of $0.1-330 \mathrm{~Hz}$, digitized at $1,000 \mathrm{~Hz}$, and stored for off-line analysis. The subjects' head position during MEG recordings was continuously monitored.

\section{6 | MRI recording}

Structural brain MRIs ( $1 \mathrm{~mm}^{3}$ T1-weighted) were obtained for all participants and used for source reconstruction.

\section{7 | MEG data preprocessing}

The data was first de-noised using the Temporal Signal-Space Separation (tSSS) method (Taulu \& Hari, 2009) and adjusted to a common head position. The de-noised data was filtered between 1 and $145 \mathrm{~Hz}$ and resampled at $500 \mathrm{~Hz}$. Independent component analysis (ICA) was used for correction of biological artifacts. The data was then epoched ( -1 to $1.2 \mathrm{~s}$ relative to the stimulus onset) and checked for the presence of residual artifacts. After rejection of the artifact-contaminated epochs and error trials, the average number of the "good" epochs for the "slow," "medium," and "fast" conditions was 79.5, 79.1, 77.1 in the $\mathrm{NT}$, and 75.4, 75.9, 76.9 in the ASD, groups. No group differences in the numbers of valid trials were found (all $p$ 's $>.3$ ). Details for the preprocessing are given in the Supporting Information Methods.

\section{8 | MEG source analysis}

The localization of the sources of gamma activity was performed with help of the FieldTrip M/EEG toolbox (Oostenveld, Fries, Maris, \& Schoffelen, 2011). To check for possible group differences in the magnitude of the visual gamma response, we first performed source localization using the DICS inverse solution algorithm (Gross et al., 2001). The frequency window was centered at the weighted peak gamma frequency (assessed at the sensor level based on dpss [Slepian sequences] multitaper analysis), with a $\pm 5 \mathrm{~Hz}$ smoothing. The significance of the group differences was then analyzed with a cluster-based permutation test (see Supporting Information Methods for details).

To assess individual peak frequencies and power ratios of the gamma response at the source maximum, we used LCMV beamformer (see Supporting Information Methods for details). For each subject and experimental condition, the weighted peak gamma power and frequency were then calculated for the average spectrum of the virtual sensors in 25 voxels closest to and including the "maximally modulated voxel" (the voxel in the visual cortex with highest increase of 45-90 Hz power during stimulation). A frequency range of interest was defined as those frequencies where the (stimulus-baseline)/ baseline power ratio exceeded $2 / 3$ of the maximum for the particular subject and condition. The gamma peak frequency was calculated as the center of gravity, whereas the gamma response power was calculated as the average power over that range. For each subject/condition, we also calculated probabilities of the post-stimulus increase in the $45-90 \mathrm{~Hz}$ gamma power in the selection of the 25 voxels. The individual peak gamma frequencies were analyzed only if the probability of the gamma power increase during stimulation period relative to pre-stimulus interval was significant at $p<.0001$.

In order to quantify the suppression of the gamma response power with increasing visual motion velocity, we introduced the "gamma suppression slope" index (GSS). We calculated the coefficient of regression of the weighted gamma response power to velocity using the "fitlm" Matlab function: fitlm $\left(x, y,{ }^{\prime} y \sim x 1-1^{\prime}\right)$; where $x=[1.2,3.6,6.0], y=[0$, $\mathrm{POW}_{\text {medium }} / \mathrm{POW}_{\text {slow }}-1, \mathrm{POW}_{\text {fast }} / \mathrm{POW}_{\text {slow }}-1$ ] and " $y \sim x 1-1$ " sets the intercept of the regression line to zero. The resulting regression coefficient $b$ is equal to zero in the case of a constant response power in the three experimental conditions (i.e., "no suppression") and is proportionally more negative in case of stronger suppression of the gamma response with increasing motion velocity (Figure 2).

The suppression of gamma response with increasing velocity can be reliably estimated only if a reliable response is observed, at least in the slow velocity condition. We estimated the GSS only if the probability of the post-stimulus gamma increase in the "slow" condition relative to pre-stimulus baseline was high $(p<.0001)$; this lead to exclusion of one ASD participant from the correlation analysis.

\section{9 | Statistical analysis}

T-test was applied to analyze group differences in the A/ASP measures. Since the distribution of some MEG parameters failed a normality test, we used Spearman coefficients for correlation analyses. The repeated measures ANOVA was initially used to test for the effects of Condition, Group, and Group $\times$ Condition interaction on the gamma parameters, and the post-hoc comparisons were performed using the nonparametric Mann-Whitney U-test.

\section{3 | RESULTS}

\section{1 | Adolescent/adult sensory profile (A/ASP)}

The general Sensory Sensitivity was marginally higher in the ASD than in NT participants $\left(T_{[37]}=2.25, p=.03\right.$ ), while no group differences were found for the visual scales (Table 1). In Supporting Information Table S1, we present the results of group comparisons for all standard A/ASP scales.

\subsection{MEG gamma responses in NT and ASD}

The cluster-based permutation test revealed no significant group differences in gamma response power in any of the three velocity conditions (all $p^{\prime} s>.08$ ). In both groups, the average location of the maximally induced voxel corresponded to the left calcarine sulcus and did not significantly differ between groups for either $x, y$, or $z$ coordinates (NT: $x=-0.18, y=-9.34, z=-0.15$; ASD: $x=0.23$, 


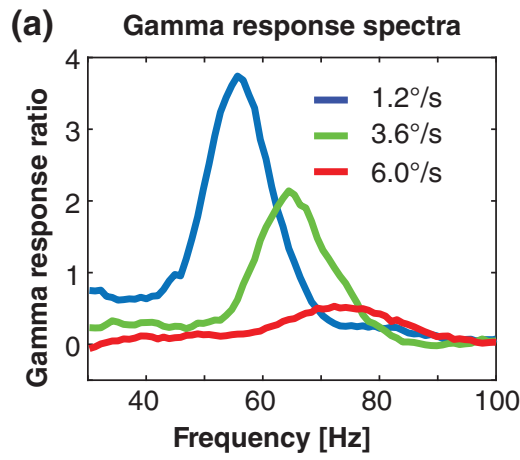

\section{Gamma suppression slope}

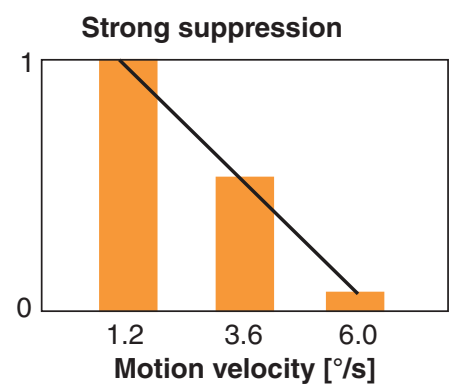

(b)
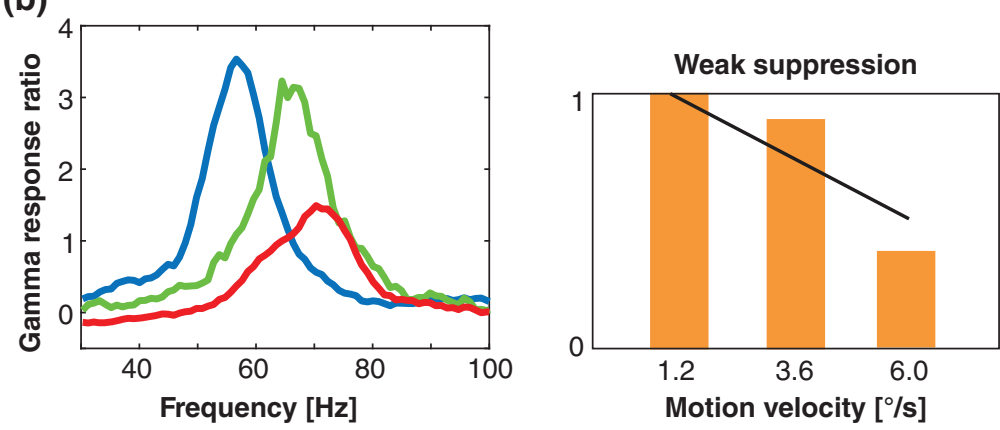

FIGURE 2 Gamma suppression slope (GSS). The left panel shows spectra of gamma power ratios: (stimulus-baseline)/baseline for two subjects. The right panel demonstrates corresponding GSSs. Subject a shows a strong suppression of gamma response power with increasing motion velocity reflected in strongly negative slope of the regression line. Subject $\mathbf{b}$ has a less prominent gamma suppression corresponding to less negative GSS value [Color figure can be viewed at wileyonlinelibrary.com]

$y=-9.49, z=0.13 \mathrm{~cm}$ ). Figure 3 shows the average source localization of the motion-related gamma response measured as the weighted peak power (see Materials and Methods for details) in the ASD and NT participants. These results indicate that in both groups, and in all experimental conditions, the gamma response predominantly reflects activity in the primary visual cortex.

For further analysis we averaged the response spectra across the maximally induced voxel and the 24 closest voxels.

Previous studies suggest that frequency of visually induced gamma oscillations might be altered in people with ASD (Dickinson, Bruyns-Haylett, Jones, \& Milne, 2015; Stroganova et al., 2012). To test for the group differences in gamma response parameters, as well as for effect of condition and its interaction with the experimental group, we used repeated measures ANOVA (rmANOVA) with the factors GROUP and VELOCITY. The power of the gamma response strongly decreased with increasing velocity $\left(F_{[2,74]}=74.2, p<.00001\right)$, but neither effect of GROUP nor GROUP $\times$ VELOCITY interaction were significant for the gamma response power ( $p$ 's $>.2$ ) (Figure 4a). To analyze the frequency of the gamma response, we measured weighted gamma response frequency in those subjects and conditions where

TABLE 1 Group differences in the A/ASP measures of sensory sensitivity

\begin{tabular}{llll} 
A/ASP item & NT (19) & ASD (20) & T-statistic \\
\hline Sensory sensitivity & 31.2 & 37.7 & $-2.3 *$ \\
\hline A/ASP-derived measures of visual sensitivity & & \\
Visual low threshold & 12.9 & 14.9 & -1.5 \\
Visual motion sensitivity & 4 & 4.7 & -1.0 \\
\hline
\end{tabular}

*Significance values $p<.05$ the stimulus-related increases in gamma response power were reliable at $p<.0001$ level (see Materials and Methods for details). The rmANOVA was performed in 15 NT and 11 ASD subjects in whom the frequency was possible to assess in each of the three velocity conditions. For the gamma frequency the rmANOVA revealed highly reliable increase in frequency with increasing motion velocity $\left(F_{[2,48]}=152.6\right.$, $p<.00001$ ), but no effect of GROUP or GROUP $\times$ VELOCITY interaction ( $p$ 's $>.5$ ) (Figure 4b). To sum up, suppression of gamma response power and increase of gamma response frequency with increasing motion velocity were observed in both NT and ASD individuals and did not differ between the groups.

\section{3 | Gamma response suppression and sensory sensitivity}

To quantify the velocity-related suppression of gamma response power, we introduced the "gamma suppression slope" (GSS) parameter (Figure 2), where a more negative GSS value corresponds to stronger suppression.

A poor signal to noise ratio (SNR), resulting from strong myogenic artifacts or low amplitude visual gamma oscillations, may reduce the gamma response power and lead to less prominent velocity-related changes, that is, less negative GSS. Because the gamma response in the "slow" condition displayed the highest power and inter-individual variability (Figure 4), one would expect that the contribution of the SNR would result in a negative correlation between GSS and the amplitude of gamma response under the "slow" condition. We, however, found that such a correlation was not significant $\left(R_{[38]}=-0.05 ; p=.78\right)$. On the other hand, the correlations of GSS with the power of the gamma 


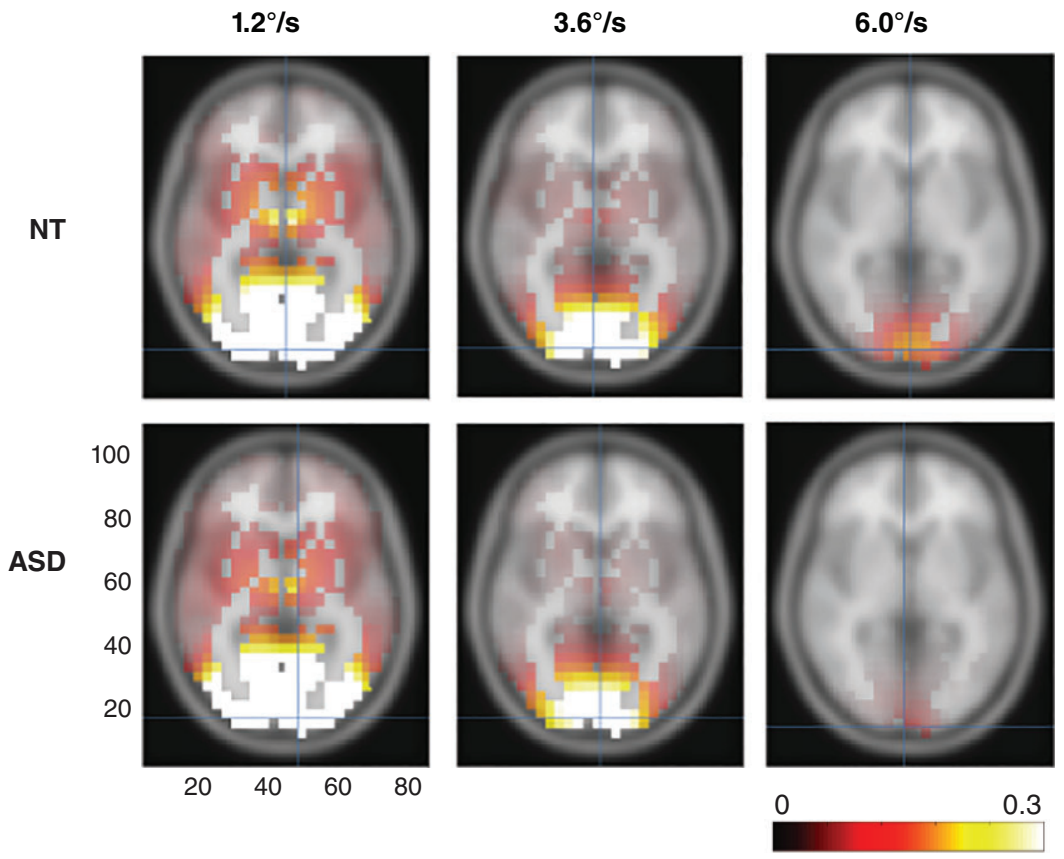

FIGURE 3 Grand average source localization of the visual gamma responses in ASD and NT individuals. Here and hereafter the magnitude of the gamma response was calculated as the ratio: (stimulus - baseline)/baseline. In both groups and under all the three velocity conditions the gamma power ratio was maximal in the calcarine sulcus (marked with a blue crosshair) [Color figure can be viewed at wileyonlinelibrary.com]

responses induced by gratings moving with medium-and especially with fast-velocities were significant (medium: $R_{(38)}=0.46, p<.01$, fast: $R_{(38)}=0.74, p<.000001$ ). This shows that a dominating contribution to GSS is not the SNR, but rather the stimulation-related suppression of the genuine gamma response.

Because the ASD and NT groups differed in neither visual sensitivity nor gamma parameters, we first combined them for the correlation analysis. Table 2 and Figure 5 show Spearman correlations between the sensitivity measures and the gamma suppression slope. As expected, less negative GSS (i.e., less prominent gamma response suppression) correlated with higher scores on Sensory Sensitivity, visual Low Threshold, and Visual Motion Sensitivity scales. The correlations remained significant when the five subjects who lacked the eye tracking data were excluded from analysis ( $R$ 's $>0.47, p^{\prime} s<.01$ ). Exclusion of the single female subjects also did not affect the results. The correlation between Sensory Sensitivity and GSS remained significant when tested separately in the NT and the ASD groups. For the

(a)

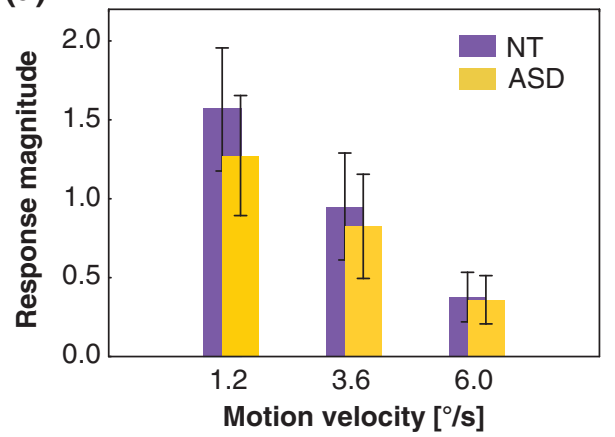

visual sensitivity measures the correlations with the GSS were in the same direction in the NT and the ASD groups, but did not reach significance level in the NT subjects. Correlations between the GSS and other A/ASP scales are presented in the Supporting Information Table S2 for comparison.

The GSS is a relational measure and its correlations with the sensory sensitivity could be predominantly driven by gamma responses at particular velocities of the visual motion. In accord with the "gain control" hypothesis the variability in gamma response strength to the most intensive stimulation ("fast" visual motion) should make major contribution to the individual variation in sensory sensitivity. Indeed, as predicted, the generally elevated visual sensitivity/avoidance and, in particular, sensitivity to visual motion were associated with higher gamma responses to the fast motion (Table 3).

Unlike the gamma suppression slope, neither the peak frequencies nor power ratios of gamma responses were reliably related to the A/ASP measures of sensitivity (all $p$ 's > .05).

(b)

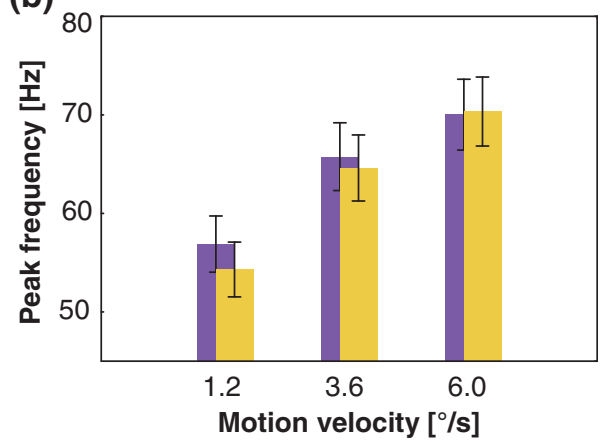

FIGURE 4 Magnitude (a) and peak frequency (b) of gamma responses to moving gratings in NT and ASD individuals. Parameters of the gamma response were measured in its focus in the visual cortex (see Materials and Methods for details). Vertical bars denote 0.95 confidence intervals [Color figure can be viewed at wileyonlinelibrary.com] 
TABLE 2 Spearman correlations between gamma suppression slope and A/ASP sensitivity measures

\begin{tabular}{|c|c|c|c|}
\hline A/ASP item & NT + ASD (38) & ASD (19) & NT (19) \\
\hline \multicolumn{4}{|l|}{ A/ASP quadrants } \\
\hline Sensory sensitivity & $0.5 * *$ & $0.57 *$ & $0.5^{*}$ \\
\hline \multicolumn{4}{|c|}{ A/ASP-derived measures of visual sensitivity } \\
\hline Visual low threshold & $0.47 * *$ & $0.62 * *$ & 0.32 \\
\hline Visual motion sensitivity & $0.51 * * *$ & $0.63 * *$ & $0.41^{\#}$ \\
\hline
\end{tabular}

Significance values $* p<.05, * * p<.01, * * * p<.001,{ }^{*} p<.1$.

\section{4 | Eyetracking}

\subsection{1 | Microsaccades}

The average number of microsaccades during the full epoch of analysis (i.e., -1 to $1.2 \mathrm{~s}$ relative to stimulus onset) was greater in ASD than in NT participants (NT: 1.43, ASD: 1.87 microsaccades per second; $\left.T_{[32]}=2.04, p<.05\right)$. The probability for microsaccade occurrence was higher in the ASD than in the NT group during fixation on the cross $\left(T_{[32]}=2.5, p<.05\right)$, but not during visual motion conditions ( $p$ 's > .06). This result is in line with a previous report on lower fixation stability in ASD (Shirama, Kanai, Kato, \& Kashino, 2016). The ANOVA with factor Interval (prestimulus, slow, medium and fast) revealed its significant effect on the number of microsaccades $\left(F_{[3,99]}=25.5\right.$, $\gamma=0.43, p<.0001)$, mainly due to a higher probability of microsaccde occurrence during prestimulus (0.044), followed by medium (0.029), slow (0.026), and fast (0.025) stimuli. Neither magnitude of the gamma responses, nor GSS, correlated with microsaccade probability in the prestimulus interval (Spearman R, all $p$ 's $>.08$ ) or during visual stimulation (all $p$ 's > .3). This result corroborates the findings of Wieczorek, who found no evidence for a sizable contribution of microsaccades to MEG gamma activity induced by visual motion (Wieczorek, 2015). There was no correlation between the SP measures (Sensory Sensitivity, Visual Low Threshold, Visual Motion Sensitivity) and microsaccade probability in either time interval (Spearman R, all $p$ 's > .12).

\subsection{2 | Fixation position}

The median value of fixation deviation from the middle position on the screen ("median deviation") was greater during the prestimulus interval than during the stimulus intervals $(F[3,99]=17.2, p<.0001$; prestimulus: $0.84^{\circ}$, slow: $0.63^{\circ}$, medium: $0.67^{\circ}$, fast: $0.68^{\circ}$ of visual angle). During the stimulus intervals, the median deviation was
TABLE 3 Spearman correlations between gamma response strength in the three velocity conditions and the a/ASP measures in the combined sample (NT + ASD)

\begin{tabular}{llll} 
& \multicolumn{3}{l}{$\begin{array}{l}\text { Gamma response magnitude: } \\
\text { (stimulus - baseline)/baseline }\end{array}$} \\
\cline { 2 - 4 } A/ASP item & Slow & Medium & Fast \\
Sensory sensitivity & $-0,23$ & 0.08 & $0.27 \#$ \\
Visual low threshold & 0.05 & 0.26 & $0.44 * *$ \\
Visual motion sensitivity & 0.03 & 0.31 & $0.46 * *$ \\
\hline
\end{tabular}

Significance values $\# p<.1, * * p<.01$.

marginally lower in ASD than in NT participants (slow: $0.57^{\circ}$ in ASD, $0.68^{\circ}$ in NT, $p=.05$; medium: $0.62^{\circ}$ in ASD, $0.72^{\circ}$ in NT, $p=.08$; fast: $0.61^{\circ}$, in ASD, $0.74^{\circ}$ in NT, $p=.02$ ). The gamma response parameters (i.e., gamma response magnitudes, GSS) did not significantly correlate with median deviations measured in either of the time intervals (i.e., prestimulus, slow, medium, fast) (all p's > .05).

\section{DISCUSSION}

Our study shows that inter-individual variations in sensory sensitivity are strongly related to the capacity to modulate MEG gamma oscillations according to intensity of the visual input. We found that subjects who reported heightened sensory sensitivity were characterized by weakened suppression of the induced gamma response with increasing velocity of visual motion. This result indicates that the neural mechanisms underlying gamma response suppression also modulate subjective reactivity to sensory events in the everyday life. Moreover, the similar pattern of findings in the NT and ASD individuals suggests that sensory hypersensitivity shares a common neural ground in people with autism and in general population. Analysis of oculomotor behavior shows that these results are unlikely to be accounted for by differences in either the number of microsaccades or fixation position.

In both NT and ASD subjects, the increase in velocity of the visual motion from 1.2 to $6 \%$ s elicited a strong and reliable suppression of the visual gamma response accompanied by a substantial increase in gamma response frequency for almost $15 \mathrm{~Hz}$ (Figure 3). These findings extend our previous results on the velocity-related changes of visual gamma response in NT subjects (Orekhova et al., 2015, 2018) and children with ASD (Stroganova et al., 2015) by replicating these findings in a group of adult ASD individuals. (a)

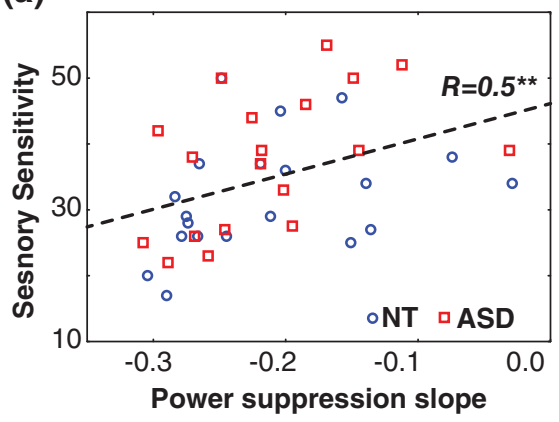

(b)

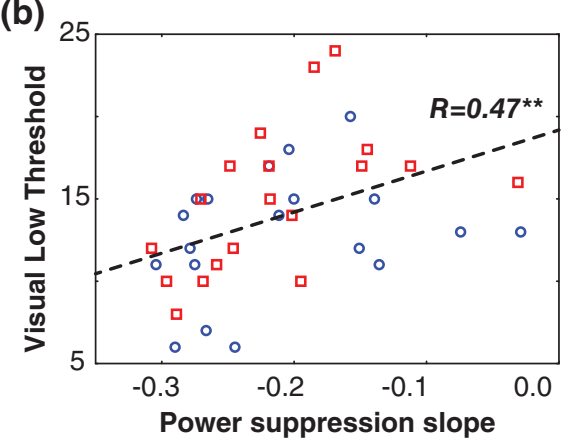

(c)

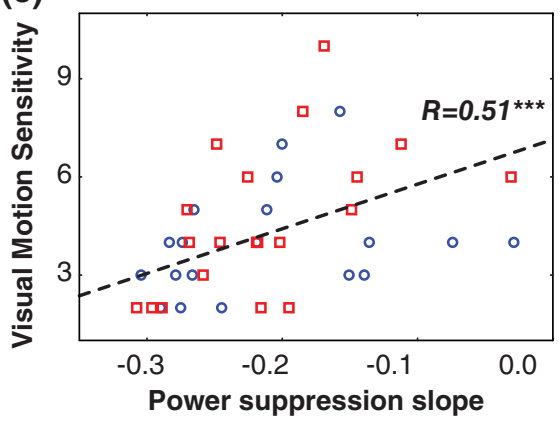

FIGURE 5 The relationship between gamma suppression slope and sensory sensitivity (a), visual low threshold (b), and visual motion sensitivity (c) in the combined sample of ASD (read squares) and NT (blue circles) individuals [Color figure can be viewed at wileyonlinelibrary.com] 
Increasing motion velocity of full-contrast visual gratings up to $6.0 \%$ s likely promotes excitation of interconnected E- and I-neurons in the visual cortical areas (see (Orekhova et al., 2018) for discussion). According to the computational modeling results of Borgers et al., increasing excitation of the I-neurons above some critical threshold leads to neuronal de-synchronization and thus suppression of gamma oscillations (Borgers \& Kopell, 2005; Borgers \& Walker, 2013; Cannon et al., 2014). This inhibitory-based physiological mechanism offers a reasonable explanation for the relative suppression of the induced gamma response at high stimulation intensities (visual motion at 3.6 and $6 \%$, Figure 4 ) in our study. Since gamma synchrony increases the impact of synaptic input from a neuronal group onto its postsynaptic targets (Fries, 2005, 2009, 2015; Ni et al., 2016; Vinck et al., 2013), the reduction of gamma power at high stimulation intensities may limit signal transmission between activated neural assemblies, thus protecting them from sensory-driven hyper-excitation. Hence, gamma suppression may reflect a suppressive gain-control mechanism, which affects sensory perception by reducing the impact of high-intensity stimulation. In a similar vein, a weaker suppression of visual gamma oscillations at the high motion velocities may be associated with a heightened behavioral sensitivity to the high-intensity visual stimulation.

To pursue this hypothesis, we introduced a measure that quantified the suppression of the gamma response with increasing stimulation intensity-the "gamma suppression slope" (GSS). As expected, a weaker negative slope (i.e., lower gamma response suppression and less efficient homeostatic regulation of the E-I balance) correlated with a higher incidence of sensory noticing/discomfort and avoidant behaviors in both the NT and the ASD individuals (Table 2 and Figure 3). Presence of similar correlation patterns in the NT and ASD subjects suggests that variations in response gain control contribute similarly to individual differences in sensory sensitivity in the ASD group and the general population. The higher correlations in the ASD individuals can be explained by somewhat greater variability of their sensory sensitivity scores comparing to those in the NT subjects. The fact that the highly sensitive individuals displayed elevated gamma responses to the high-intensity stimuli, rather than reduced responses to those at lower intensities (Table 3), gives additional support for the suggested link between sensory hypersensitivity and inefficient down-regulation of excessive activation of the visual cortex. In general, these results confirm our hypothesis that the mechanisms leading to gamma response suppression in case of strong sensory input serve to protect the brain from hyper-excitation.

Most probably, the suppression of the gamma response at the highest stimulus velocity/temporal frequency used in our study $(6 \% \mathrm{~s}$ or $10 \mathrm{~Hz}$ ) is associated mainly with decrease in the efficacy of transient interactions between neural populations rather than with reduction of neuronal firing. Indeed, a recent study in monkeys showed that the $50-80 \mathrm{~Hz}$ power in local field potential (LFP) recordings reaches its maximum at lower temporal frequencies of visual motion than the neuronal spiking does (Salelkar et al., 2018). In particular, when the temporal frequency of visual motion increased from 2 to $8 \mathrm{~Hz}$ the suppression of the LFP gamma response in monkeys was paralleled by an increase or absence of change in neuronal firing. Similarly, in humans, BOLD activation in visual cortical areas elicited by drifting visual gratings drops only after increasing temporal frequency beyond $9 \mathrm{~Hz}$ (Singh, Smith, \& Greenlee, 2000), suggesting high level of cortical activation at this temporal frequency. According to the model of Borgers and Kopell (2005), the asynchronous activity of the over-excited I-neurons, corresponding to the "no gamma state," can suppress activity of the E-neurons. It is therefore likely that motion velocities/temporal frequencies yet higher than those applied in our study would result in a complete blockage of gamma oscillations paralleled by a decrease in E-neurons firing in the visual cortex.

Given that the homeostatic control of neural excitability may differ between cortical areas, we expected that the suppression of visual gamma response would be most closely related to behavioral sensitivity in the visual domain. Indeed, in case of the "Low Threshold" A/ASP measures, the correlation with the GSS was highest for the visual modality (Supporting Information Table S2). Correlations with the GSS in nonvisual sensory or behavioral domains can be explained by presence of common neural factors affecting response gain control across sensory modalities, that is, global variations in neural excitability, functional or structural connectivity, etc.

It is noteworthy that the majority of the ASD and NT participants in our study differed neither in regard to visual sensitivity (Table 1), nor gamma response parameters (Figure 2). These results suggest that the capacity to down-regulate growing neural excitation with increasing intensity of a sensory input was relatively preserved in the visual cortex in our high-functioning adult participants with ASD. Although an altered $\mathrm{E}-\mathrm{I}$ balance is thought to be an important mechanism of ASD (Levin \& Nelson, 2015; Rubenstein \& Merzenich, 2003), it is possible that it is less affected (or better compensated for Nelson \& Valakh, 2015) in the visual cortex than in other cortical areas. For example, Gaetz et al. (2014) reported that the concentration of the inhibitory transmitter gamma-aminobutyric acid (GABA) was normal in the visual cortex of adolescents with ASD, while being significantly reduced in their auditory and motor cortices. Yet another possibility is that our participants with ASD represented a subgroup characterized by a relatively low prevalence of atypical sensory sensitivity, including that in the visual domain, compared with a more general ASD population. For example, in the study of Crane et al, the Sensory Sensitivity scores in adults with ASD were higher than in our study (Crane et al: 45.0; this study: 37.7), while the corresponding scores for the NT subjects were more similar (Crane et al: 33.8; this study: 31.2).

Recent advances in genetics and neuroscience clearly demonstrate that behavioral symptoms of ASD and other neurodevelopmental disorders may stem from cardinally different genetic and molecular etiologies that cause either increases or decreases in the $\mathrm{E}-\mathrm{I}$ ratio (Lee et al., 2017; Nelson \& Valakh, 2015). Given the heterogeneous nature of ASD (Gillberg, 2010; Jeste \& Geschwind, 2014; Tordjman et al., 2018), the abnormal capacity to regulate the E-I balance in the visual cortex could characterize only a proportion of ASD individuals, as well as, for example, patients with fragile $X$ syndrome who are often hypersensitive to visual stimuli and are suggested to have elevated neural excitability of the visual cortex (Rigoulot et al., 2017; Schneider, Hagerman, \& Hessl, 2009; Sinclair, Oranje, Razak, Siegel, \& Schmid, 2017; Van der Molen et al., 2012). Considering the reliable correlation between sensory sensitivity and GSS (Figure 5), one may predict that these individuals would demonstrate reduced suppression of visual 
gamma response. In this respect, the GSS measure may provide a biomarker that can be used to select ASD subgroups according to a distinct neural phenotype-E-I imbalance in the visual cortex. Considering that many promising pharmacological agents tested or being testing in the ASD in clinical trials target the E-I balance, stratification of this clinical population according to the relevant neural deficits is important for selection of an individually appropriate treatment and tracking the treatment outcome.

Our study has several limitations. First, our participants were nearly exclusively males. An additional study is needed to generalize the results to females. Second, each of the experimental groups had a relatively small sample size, which stresses the need for an independent replication study. Third, although our participants with ASD had marginally higher sensory responsiveness than the NT individuals, there was large overlap between the groups (Figure 5). It would be important in the future to investigate gamma response suppression in individuals characterized by excessive hypersensitivity, particularly in visual modality. Fourth, our experimental paradigm was specifically aimed at testing the gain control in the visual cortex, and it is unclear whether a similar modulation of cortical gamma responses by input intensity is present in other sensory modalities, for example, auditory or tactile.

In conclusion, the modulation of gamma response power by intensity of visual input may give important information about the neural mechanisms that mitigate rising excitation and maintain E-I balance in the visual networks. Given the need for sensitive and objective measures of region-specific cortical excitability in different patient populations, this input-output relationship in gamma response strength offers a promising translational tool for clinical research. We suggest that the slope of the stimulus-response function of visual gamma may provide a tractable and accessible measure of the capacity to regulate the E-I balance in visual circuitry according to intensity of the visual input. It could be an especially appropriate measure in some groups of patients characterized by an elevated cortical excitability and high sensitivity to visual stimuli, such as patients with photo-sensitive epilepsy, migraine, and some forms of ASD. This noninvasive biomarker for unbalanced cortical excitability could also be used to select distinct sub-groups of patients within heterogeneous clinical populations (e.g., within ASD) and to track the impact of tailored pharmacological interventions in clinical trials.

\section{ACKNOWLEDGMENTS}

This work was financed by the Torsten Soderberg Foundation (M240/13 to CG), the Russian Scientific Foundation (14-35-00060 to TS). Authors JFS and BR are supported by the Knut and Alice Wallenberg Foundation (grant 2014.0102), the Swedish Research Council (grant 621-2012-3673), and the Swedish Childhood Cancer Foundation (grant MT2014-0007). The author NH was supported by the LifeWatch Foundation. The author TS was supported by the Charity Foundation "Way Out". The study was partly supported by the HSE Basic Research Program and the Russian Academic Excellence Project "5-100". We heartily thank all participants for their participation in this study.

\section{CONFLICT OF INTEREST}

The authors declare that they have no conflicts of interest.

\section{ORCID}

Elena V. Orekhova (D) https://orcid.org/0000-0003-0950-1613 Justin F. Schneiderman (D) https://orcid.org/0000-0002-4441-2360 Nouchine Hadjikhani (D) https://orcid.org/0000-0003-4075-3106

\section{REFERENCES}

Bargary, G., Furlan, M., Raynham, P. J., Barbur, J. L., \& Smith, A. T. (2015). Cortical hyperexcitability and sensitivity to discomfort glare. Neuropsychologia, 69, 194-200. https://doi.org/10.1016/j.neuropsychologia. 2015.02.006

Borgers, C., \& Kopell, N. (2005). Effects of noisy drive on rhythms in networks of excitatory and inhibitory neurons. Neural Computation, 17, 557-608. https://doi.org/10.1162/0899766053019908

Borgers, C., \& Walker, B. (2013). Toggling between gamma-frequency activity and suppression of cell assemblies. Frontiers in Computational Neuroscience, 7, 33. https://doi.org/10.3389/fncom.2013.00033

Boulloche, N., Denuelle, M., Payoux, P., Fabre, N., Trotter, Y., \& Geraud, G. (2010). Photophobia in migraine: An interictal PET study of cortical hyperexcitability and its modulation by pain. Journal of Neurology Neurosurgery and Psychiatry, 81, 978-984. https://doi.org/10.1136/jnnp. 2009.190223

Brown, C. E., \& Dunn, W. (2002). Adolescent/adult sensory profile: User's manual. San Antonio, TX: The Psychological Corporation.

Buzsaki, G., \& Wang, X. J. (2012). Mechanisms of gamma oscillations. Annual Review of Neuroscience, 35, 203-225. https://doi.org/10.1146/ annurev-neuro-062111-150444

Cannon, J., McCarthy, M. M., Lee, S., Lee, J., Borgers, C., Whittington, M. A., \& Kopell, N. (2014). Neurosystems: Brain rhythms and cognitive processing. The European Journal of Neuroscience, 39, 705-719. https://doi.org/10.1111/ejn.12453

Cousijn, H., Haegens, S., Wallis, G., Near, J., Stokes, M. G., Harrison, P. J., \& Nobre, A. C. (2014). Resting GABA and glutamate concentrations do not predict visual gamma frequency or amplitude. Proceedings of the National Academy of Sciences of the United States of America, 111, 9301-9306. https://doi.org/10.1073/pnas.1321072111

Davidsson, M., Hult, N., Gillberg, C., Sarneo, C., Gillberg, C., \& Billstedt, E. (2017). Anxiety and depression in adolescents with ADHD and autism spectrum disorders; correlation between parent- and self-reports and with attention and adaptive functioning. Nordic Journal of Psychiatry, 71, 614-620. https://doi.org/10.1080/08039488.2017.1367840

Dehghani, N., Peyrache, A., Telenczuk, B., Le Van Quyen, M., Halgren, E., Cash, S. S., ... Destexhe, A. (2016). Dynamic balance of excitation and inhibition in human and monkey neocortex. Scientific Reports, 6(1), 23176. https://doi.org/10.1038/srep23176

Dickinson, A., Bruyns-Haylett, M., Jones, M., \& Milne, E. (2015). Increased peak gamma frequency in individuals with higher levels of autistic traits. The European Journal of Neuroscience, 41, 1095-1101. https:// doi.org/10.1111/ejn.12881

Dorrn, A. L., Yuan, K., Barker, A. J., Schreiner, C. E., \& Froemke, R. C. (2010). Developmental sensory experience balances cortical excitation and inhibition. Nature, 465, 932-937. https://doi.org/10.1038/ nature09119

Ecker, C., Spooren, W., \& Murphy, D. G. M. (2013). Translational approaches to the biology of autism: False dawn or a new era? Molecular Psychiatry, 18, 435-442. https://doi.org/10.1038/mp.2012.102

Edden, R. A., Muthukumaraswamy, S. D., Freeman, T. C., \& Singh, K. D. (2009). Orientation discrimination performance is predicted by GABA concentration and gamma oscillation frequency in human primary visual cortex. The Journal of Neuroscience, 29, 15721-15726. https:// doi.org/10.1523/JNEUROSCI.4426-09.2009

Fries, P. (2005). A mechanism for cognitive dynamics: Neuronal communication through neuronal coherence. Trends in Cognitive Sciences, 9, 474-480. https://doi.org/10.1016/j.tics.2005.08.011 
Fries, P. (2009). Neuronal gamma-band synchronization as a fundamental process in cortical computation. Annual Review of Neuroscience, 32, 209-224. https://doi.org/10.1146/annurev.neuro.051508.135603

Fries, P. (2015). Rhythms for cognition: Communication through coherence. Neuron, 88, 220-235. https://doi.org/10.1016/j.neuron.2015. 09.034

Gaetz, W., Bloy, L., Wang, D. J., Port, R. G., Blaskey, L., Levy, S. E., \& Roberts, T. P. (2014). GABA estimation in the brains of children on the autism spectrum: Measurement precision and regional cortical variation. Neuroimage, 86, 1-9. https://doi.org/10.1016/j.neuroimage.2013. 05.068

Gillberg, C. (2010). The ESSENCE in child psychiatry: Early symptomatic syndromes eliciting neurodevelopmental clinical examinations. Research in Developmental Disabilities, 31, 1543-1551. https://doi. org/10.1016/j.ridd.2010.06.002

Gross, J., Kujala, J., Hamalainen, M., Timmermann, L., Schnitzler, A., \& Salmelin, R. (2001). Dynamic imaging of coherent sources: Studying neural interactions in the human brain. Proceedings of the National Academy of Sciences of the United States of America, 98, 694-699. https://doi.org/10.1073/pnas.98.2.694

Haigh, S. M., Barningham, L., Berntsen, M., Coutts, L. V., Hobbs, E. S., Irabor, J., ... Wilkins, A. J. (2013). Discomfort and the cortical haemodynamic response to coloured gratings. Vision Research, 89, 47-53. https://doi.org/10.1016/j.visres.2013.07.003

Helles, A., Gillberg, C. I., Gillberg, C., \& Billstedt, E. (2015). Asperger syndrome in males over two decades: Stability and predictors of diagnosis. Journal of Child Psychology and Psychiatry, 56, 711-718. https://doi. org/10.1111/jcpp.12334

Horder, J., Wilson, C. E., Mendez, M. A., \& Murphy, D. G. (2014). Autistic traits and abnormal sensory experiences in adults. Journal of Autism and Developmental Disorders, 44, 1461-1469. https://doi.org/10. 1007/s10803-013-2012-7

Isaacson, J. S., \& Scanziani, M. (2011). How inhibition shapes cortical activity. Neuron, 72, 231-243. https://doi.org/10.1016/j.neuron.2011.09.027

Jeste, S. S., \& Geschwind, D. H. (2014). Disentangling the heterogeneity of autism spectrum disorder through genetic findings. Nature Reviews. Neurology, 10, 74-81. https://doi.org/10.1038/nrneurol.2013.278

Jia, X. X., Smith, M. A., \& Kohn, A. (2011). Stimulus selectivity and spatial coherence of gamma components of the local field potential. The Journal of Neuroscience, 31, 9390-9403. https://doi.org/10.1523/ Jneurosci.0645-11.2011

Jia, X. X., Xing, D. J., \& Kohn, A. (2013). No consistent relationship between gamma power and peak frequency in macaque primary visual cortex. The Journal of Neuroscience, 33, U417-U421. https://doi. org/10.1523/Jneurosci.1687-12.2013

LeBlanc, J. J., \& Fagiolini, M. (2011). Autism: A "critical period" disorder? Neural Plasticity, 1-17, document number 921680 . https://doi.org/10.1155/ 2011/921680

Lee, E., Lee, J., \& Kim, E. (2017). Excitation/inhibition imbalance in animal models of autism Spectrum disorders. Biological Psychiatry, 81 838-847. https://doi.org/10.1016/j.biopsych.2016.05.011

Levin, A. R., \& Nelson, C. A. (2015). Inhibition-based biomarkers for autism Spectrum disorder. Neurotherapeutics, 12, 546-552. https://doi. org/10.1007/s13311-015-0350-1

Little, L. M., Dean, E., Tomchek, S. D., \& Dunn, W. (2017). Classifying sensory profiles of children in the general population. Child: Care, Health and Development, 43, 81-88. https://doi.org/10.1111/cch.12391

Maniyar, F. H., Sprenger, T., Schankin, C., \& Goadsby, P. J. (2014). Photic hypersensitivity in the premonitory phase of migraine - a positron emission tomography study. European Journal of Neurology, 21, 1178-1183. https://doi.org/10.1111/ene.12451

Nelson, S. B., \& Valakh, V. (2015). Excitatory/inhibitory balance and circuit homeostasis in autism spectrum disorders. Neuron, 87, 684-698. https://doi.org/10.1016/j.neuron.2015.07.033

Ni, J. G., Wunderle, T., Lewis, C. M., Desimone, R., Diester, I., \& Fries, P. (2016). Gamma-rhythmic gain modulation. Neuron, 92, 240-251. https://doi.org/10.1016/j.neuron.2016.09.003

O'Hare, L., \& Hibbard, P. B. (2016). Visual processing in migraine. Cephalalgia, 36, 1057-1076. https://doi.org/10.1177/0333102415618952

Oostenveld, R., Fries, P., Maris, E., \& Schoffelen, J. M. (2011). FieldTrip: Open source software for advanced analysis of MEG, EEG, and invasive electrophysiological data. Computational Intelligence and Neuroscience, 2011, 156869, 1-9. https://doi.org/10.1155/2011/156869

Orekhova, E. V., Butorina, A. V., Sysoeva, O. V., Prokofyev, A. O., Nikolaeva, A. Y., \& Stroganova, T. A. (2015). Frequency of gamma oscillations in humans is modulated by velocity of visual motion. Journal of Neurophysiology, 114, 244-255. https://doi.org/10.1152/jn.00232. 2015

Orekhova, E. V., Sysoeva, O. V., Schneiderman, J. F., Lundstrom, S., Galuta, I. A., Goiaeva, D. E., ... Stroganova, T. A. (2018). Inputdependent modulation of MEG gamma oscillations reflects gain control in the visual cortex. Scientific Reports, 8, 8451. https://doi.org/10. 1038/s41598-018-26779-6

Peirce, J. W. (2007). The potential importance of saturating and supersaturating contrast response functions in visual cortex. Journal of Vision, 7, 13. https://doi.org/10.1167/7.6.13

Perry, G., Brindley, L. M., Muthukumaraswamy, S. D., Singh, K. D., \& Hamandi, K. (2014). Evidence for increased visual gamma responses in photosensitive epilepsy. Epilepsy Research, 108, 1076-1086. https:// doi.org/10.1016/j.eplepsyres.2014.04.012

Rigoulot, S., Knoth, I. S., Lafontaine, M. P., Vannasing, P., Major, P., Jacquemont, S., ... Lippe, S. (2017). Altered visual repetition suppression in fragile $X$ syndrome: New evidence from ERPs and oscillatory activity. International Journal of Developmental Neuroscience, 59, 52-59. https://doi.org/10.1016/j.ijdevneu.2017.03.008

Roberts, M. J., Lowet, E., Brunet, N. M., Ter Wal, M., Tiesinga, P., Fries, P., \& De Weerd, P. (2013). Robust gamma coherence between macaque $\mathrm{V} 1$ and $\mathrm{V} 2$ by dynamic frequency matching. Neuron, 78, 523-536. https://doi.org/10.1016/j.neuron.2013.03.003

Robertson, A. E., \& Simmons, D. R. (2013). The relationship between sensory sensitivity and autistic traits in the general population. Journal of Autism and Developmental Disorders, 43, 775-784. https://doi.org/10. 1007/s10803-012-1608-7

Rubenstein, J. L. R., \& Merzenich, M. M. (2003). Model of autism: Increased ratio of excitation/inhibition in key neural systems. Genes, Brain, and Behavior, 2, 255-267. https://doi.org/10.1046/j. 1601-183X.2003.00037.x

Salelkar, S., Somasekhar, G. M., \& Ray, S. (2018). Distinct frequency bands in the local field potential are differently tuned to stimulus drift rate. Journal of Neurophysiology, 120, 681-692. https://doi.org/10.1152/jn. 00807.2017

Schneider, A., Hagerman, R. J., \& Hessl, D. (2009). Fragile X syndromefrom genes to cognition. Developmental Disabilities Research Reviews, 15, 333-342. https://doi.org/10.1002/ddrr.80

Shirama, A., Kanai, C., Kato, N., \& Kashino, M. (2016). Ocular fixation abnormality in patients with autism spectrum disorder. The Journal of Autism and Developmental Disorders, 46, 1613-1622. https://doi. org/10.1007/s10803-015-2688-y

Sinclair, D., Oranje, B., Razak, K. A., Siegel, S. J., \& Schmid, S. (2017). Sensory processing in autism spectrum disorders and fragile $\mathrm{X}$ syndromefrom the clinic to animal models. Neuroscience \& Biobehavioral Reviews, 76, 235-253. https://doi.org/10.1016/j.neubiorev.2016.05.029

Singh, K. D., Smith, A. T., \& Greenlee, M. W. (2000). Spatiotemporal frequency and direction sensitivities of human visual areas measured using fMRI. Neurolmage, 12, 550-564. https://doi.org/10.1006/nimg. 2000.0642

Stroganova, T. A., Butorina, A. V., Sysoeva, O. V., Prokofyev, A. O., Nikolaeva, A. Y., Tsetlin, M. M., \& Orekhova, E. V. (2015). Altered modulation of gamma oscillation frequency by speed of visual motion in children with autism spectrum disorders. Journal of Neurodevelopmental Disorders, 7:1, 21. https://doi.org/10.1186/s11689-015-9121-x.

Stroganova, T. A., Orekhova, E. V., Prokofyev, A. O., Tsetlin, M. M., Gratchev, V. V., Morozov, A. A., \& Obukhov, Y. V. (2012). Highfrequency oscillatory response to illusory contour in typically developing boys and boys with autism spectrum disorders. Cortex, 48 , 701-717. https://doi.org/10.1016/j.cortex.2011.02.016

Taulu, S., \& Hari, R. (2009). Removal of magnetoencephalographic artifacts with temporal signal-space separation: Demonstration with single-trial auditory-evoked responses. Human Brain Mapping, 30, 1524-1534. https://doi.org/10.1002/hbm.20627

Taylor, M. J., Gustafsson, P., Larsson, H., Gillberg, C., Lundström, S., \& Lichstenstein, P. (2018). Examining the association between autistic 
traits and atypical sensory reactivity: A twin study. The Journal of the American Academy of Child and Adolescent Psychiatry, 57, 96-102. https://doi.org/10.1016/j.jaac.2017.11.019

Tordjman, S., Cohen, D., Anderson, G. M., Botbole, M., Canitano, R., Coulon, N., \& Roubertoux, P. L. (2018). Repint of "reframing autism as a behavioral syndrome and not a specific mental disorder: Implications of genetic and phenotypic heterogeneity". Neuroscience and Biobehavioral Reviews, 89, 132-150. https://doi.org/10.1016/j.neubiorev.2017.01.030

Tu, S. C., Akhtar, M. W., Escorihuela, R. M., Amador-Arjona, A., Swarup, V., Parker, J., ... Nakanishi, N. (2017). NitroSynapsin therapy for a mouse MEF2C haploinsufficiency model of human autism. Nature Communications, 8, 1488. https://doi.org/10.1038/s41467-017-01563-8

Van Campen, J. S., Jansen, F. E., Kleinrensink, N. J., Joels, M., Braun, K. P. J., \& Bruining, H. (2015). Sensory modulation disorders in childhood epilepsy. The Journal of Neurodevelopmental Disorders, 7, 34. https://doi.org/10.1186/s11689-015-9130-9

Van der Molen, M. J., Van der Molen, M. W., Ridderinkhof, K. R., Hamel, B. C., Curfs, L. M., \& Ramakers, G. J. (2012). Auditory and visual cortical activity during selective attention in fragile $X$ syndrome: $A$ cascade of processing deficiencies. Clinical Neurophysiology, 123, 720-729. https://doi.org/10.1016/j.clinph.2011.08.023

Vinck, M., Womelsdorf, T., \& Fries, P. (2013). Gamma-band synchronization and information transmission. In R. Q. Quiroga \& S. Panzeri (Eds.), Principles of neural coding (pp. 449-469). Boca Raton, FL: CRC Press.

Wieczorek, K. (2015) Investigating the relationship between microsaccades and oscillations in the human visual cortex (PhD Thesis). Cardiff University.
Xue, M. S., Atallah, B. V., \& Scanziani, M. (2014). Equalizing excitationinhibition ratios across visual cortical neurons. Nature, 511, 596-600. https://doi.org/10.1038/nature13321

Yizhar, O., Fenno, L. E., Prigge, M., Schneider, F., Davidson, T. J., O'Shea, D. J., ... Deisseroth, K. (2011). Neocortical excitation/inhibition balance in information processing and social dysfunction. Nature, 477, 171-178. https://doi.org/10.1038/nature10360

Yuval-Greenberg, S., Tomer, O., Keren, A. S., Nelken, I., \& Deouell, L. Y. (2008). Transient induced gamma-band response in EEG as a manifestation of miniature saccades. Neuron, 58, 429-441. https://doi.org/10. 1016/j.neuron.2008.03.027

\section{SUPPORTING INFORMATION}

Additional supporting information may be found online in the Supporting Information section at the end of the article.

How to cite this article: Orekhova EV, Stroganova TA, Schneiderman JF, et al. Neural gain control measured through cortical gamma oscillations is associated with sensory sensitivity. Hum Brain Mapp. 2019;40:1583-1593. https://doi.org/10. $\underline{1002 / \mathrm{hbm} .24469}$ 